\title{
Plasma parameters of pulsed-dc discharges in methane used to deposit diamondlike carbon films
}

\author{
C. Corbella, ${ }^{\text {a) }}$ M. Rubio-Roy, E. Bertran, and J. L. Andújar \\ FEMAN Group, IN $N^{2} U B$, Departament de Física Aplicada i Òptica, Universitat de Barcelona, c/Martí i \\ Franquès 1, E-08028 Barcelona, Spain
}

(Received 4 February 2009; accepted 24 June 2009; published online 5 August 2009)

\begin{abstract}
Here we approximate the plasma kinetics responsible for diamondlike carbon (DLC) depositions that result from pulsed-dc discharges. The DLC films were deposited at room temperature by plasma-enhanced chemical vapor deposition (PECVD) in a methane $\left(\mathrm{CH}_{4}\right)$ atmosphere at $10 \mathrm{~Pa}$. We compared the plasma characteristics of asymmetric bipolar pulsed-dc discharges at $100 \mathrm{kHz}$ to those produced by a radio frequency (rf) source. The electrical discharges were monitored by a computer-controlled Langmuir probe operating in time-resolved mode. The acquisition system provided the intensity-voltage $(I-V)$ characteristics with a time resolution of $1 \mu \mathrm{s}$. This facilitated the discussion of the variation in plasma parameters within a pulse cycle as a function of the pulse waveform and the peak voltage. The electron distribution was clearly divided into high- and low-energy Maxwellian populations of electrons (a bi-Maxwellian population) at the beginning of the negative voltage region of the pulse. We ascribe this to intense stochastic heating due to the rapid advancing of the sheath edge. The hot population had an electron temperature $T_{e}^{\text {hot }}$ of over $10 \mathrm{eV}$ and an initial low density $n_{e}^{\text {hot }}$ which decreased to zero. Cold electrons of temperature $T_{e}^{\text {cold }} \sim 1 \mathrm{eV}$ represented the majority of each discharge. The density of cold electrons $n_{e}^{\text {cold }}$ showed a monotonic increase over time within the negative pulse, peaking at almost $7 \times 10^{10} \mathrm{~cm}^{-3}$, corresponding to the cooling of the hot electrons. The plasma potential $V_{p}$ of $\sim 30 \mathrm{~V}$ underwent a smooth increase during the pulse and fell at the end of the negative region. Different rates of $\mathrm{CH}_{4}$ conversion were calculated from the DLC deposition rate. These were explained in terms of the specific activation energy $E_{a}$ and the conversion factor $x_{\text {dep }}$ associated with the plasma processes. The work deepens our understanding of the advantages of using pulsed power supplies for the PECVD of hard metallic and protective coatings for industrial applications (optics, biomedicine, and electronics). (C) 2009 American Institute of Physics. [DOI: 10.1063/1.3183945]
\end{abstract}

\section{INTRODUCTION}

Plasma technology is fundamental for the deposition and processing of hard coatings. ${ }^{1,2}$ Nowadays, magnetron sputtering and PECVD are the most popular industrial plasma coating methods. ${ }^{3,4}$ Pulsed-dc technology has led to the design of cost-effective deposition systems and to improved film properties, compared to previous rf systems. ${ }^{5,6}$ Besides reducing powder formation and allowing more precise control of the generation of reactive species, the use of pulsed-dc power has led to the possibility of large-scale plasma deposition processes. This is a very important issue for industry, as it offers the possibility of coating large areas and increasing the deposition throughput, ${ }^{7,8}$ especially in the production of polymers, ${ }^{9,10}$ flat panel displays, ${ }^{11,12}$ and both functional and protective coatings. ${ }^{13-17}$ Moreover, this technology has permitted the development of more sophisticated equipment with additional ionization sources ${ }^{18,19}$ and has led to the production of innovative materials. Specifically, the use of an asymmetric bipolar pulsed-dc power source operating between 50 and $350 \mathrm{kHz}$ is advantageous for the effective PECVD of DLC films. DLC contains an amorphous network of $s p^{2}$ - and $s p^{3}$-bonded carbon atoms, which confers me-

\footnotetext{
${ }^{a)}$ Author to whom correspondence should be addressed. Electronic mail: corbella@ub.edu.
}

chanical and tribological properties similar to those of diamond, such as hardness, resistance to wear, and a low friction coefficient. ${ }^{20-23}$

Thanks to pulsed power supplies, the PECVD of DLC films has achieved higher growth rates and lower stress levels. ${ }^{24}$ The waveform of the signal driven to the cathode and also the power input into the glow discharge $W$ have a strong influence on the properties of the growing film. The energy of the ions impinging onto the DLC film has a decisive influence on its structure and hydrogen content. ${ }^{21,25}$ Whereas in the case of a rf power source the maximal ion energy is limited by the self-bias potential $U_{\mathrm{sb}}$, pulsed-dc plasmas provide local peak voltages with amplitudes so large that they result in a much more intense and energetic ion bombardment. The use of pulses has also beneficial effects when growing DLC and metal-containing DLC films by pulsed-dc reactive magnetron sputtering. ${ }^{26,27}$

Used in parallel with film deposition, plasma diagnostics ensure process control and reproducibility of the process. Optical emission spectroscopy (OES), ${ }^{28,29}$ quadrupole mass spectrometry (QMS), ${ }^{30,31}$ and diagnostics by means of a retarding field energy analyzer (RFEA) $)^{32}$ and a Langmuir probe $^{33-35}$ are the most common plasma characterization techniques. The last of these provides reliable monitoring of plasma parameters by means of recording $I-V$ characteristics 
from an electrostatic tip in the glow discharge. However, the evaluation of these parameters in nonstationary processes requires the acquisition of $I-V$ curves by Langmuir probes configured in a time-resolved mode. Much progress has been reported concerning time-resolved tracking of electron temperature, plasma density, and potentials in pulsed plasmas. ${ }^{36-38}$ Such analysis increases our understanding of the plasma kinetics in discharges with time-varying patterns and involving a huge variety of gases. Hydrocarbons are the main precursors for the synthesis of DLC films: propane $\left(\mathrm{C}_{3} \mathrm{H}_{8}\right),{ }^{39}$ benzene $\left(\mathrm{C}_{6} \mathrm{H}_{6}\right),{ }^{40}$ acetylene $\left(\mathrm{C}_{2} \mathrm{H}_{2}\right)$, and $\mathrm{CH}_{4}$ are of particular importance. ${ }^{21,41-43}$ Although $\mathrm{C}_{2} \mathrm{H}_{2}$ produces harder films and faster deposition, $\mathrm{CH}_{4}$ is commonly used to coat electronic devices and is less harmful. ${ }^{21}$ The precursor can be mixed with other gases such as argon (Ar) and hydrogen $\left(\mathrm{H}_{2}\right)$ in order to modify the plasma chemistry and the surface morphology.

In this paper, the deposition of DLC in a pure $\mathrm{CH}_{4}$ atmosphere at $10 \mathrm{~Pa}$ was performed by $100 \mathrm{kHz}$ pulsed-dc PECVD and monitored by means of a fast Langmuir probe. The plasma parameters were calculated at $1 \mu$ s interval, and their evolution within the pulse cycle and against $W$ is discussed. We further analyze the heating mechanisms in the discharge and the effect of $W$ on both plasma kinetics and the deposition rate. rf discharges were also studied and compared to the results from pulsed-dc plasmas.

\section{EXPERIMENTAL SETUP}

\section{A. Reactor description and operating conditions}

A series of DLC films ranging between 200 and $1500 \mathrm{~nm}$ thickness was grown by PECVD using either a rf (13.56 $\mathrm{MHz})$ or a pulsed-dc $(100 \mathrm{kHz})$ source in a $\mathrm{CH}_{4}$ atmosphere. The deposition system included a load-lock chamber and a computer-controlled gas supply. ${ }^{24}$ The reactor consisted of a cylindrical vessel of $30 \mathrm{~cm}$ diameter and $19.5 \mathrm{~cm}$ length, where the cathode $\left(70 \mathrm{~cm}^{2}\right)$ and the anode $\left(400 \mathrm{~cm}^{2}\right)$ were placed vertically and $4 \mathrm{~cm}$ apart in a capacitive configuration. Monocrystalline silicon wafers were used as substrates. They were placed on the cathode and were kept at room temperature by means of a water cooling circuit. The $\mathrm{CH}_{4}$ flow was set to 30 SCCM (SCCM denotes cubic centimeter per minute at STP) downstream along the interelectrode gap using a mass flow controller, and it was evacuated with a Roots and a rotary pump. All the processes were carried out at a working pressure $p$ of $10 \mathrm{~Pa}$, and a base pressure of $10^{-4} \mathrm{~Pa}$ was attained with a turbomolecular pumping system. Film thickness was measured by a Dektak 3030 profilometer.

An asymmetric bipolar pulsed-dc signal was driven to the substrate holder by means of an ENI-RPG-50 power supply operated in power regulation mode. The pulse frequency was fixed at $100 \mathrm{kHz}$. Figure 1 shows the waveform of the pulsed signal as registered by a high-voltage probe connected to an oscilloscope. The waveform consisted of a fixed positive voltage of around $50 \mathrm{~V}$ after an initial overshoot of over $200 \mathrm{~V}$, followed by a variable negative pulse whose peak amplitude ranged between -700 and $-1400 \mathrm{~V}$. The signal consisted of two regions, the on and off phases, which

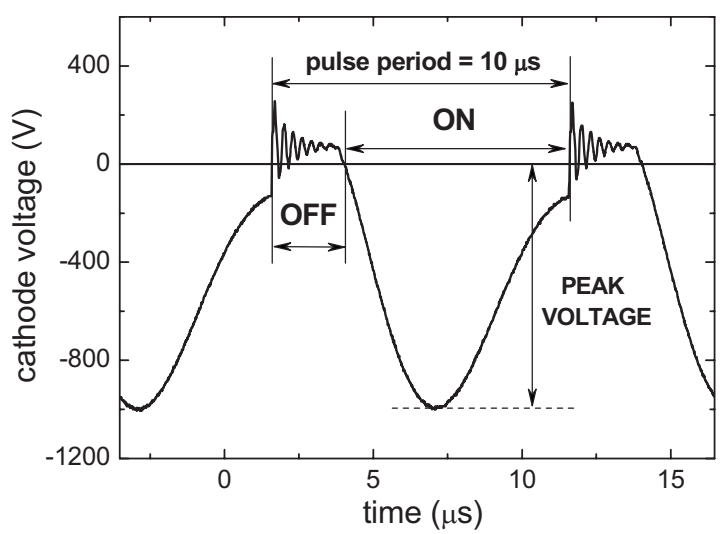

FIG. 1. Asymmetric bipolar pulsed-dc waveform used to excite a $\mathrm{CH}_{4}$ atmosphere at $10 \mathrm{~Pa}$. The pulse is divided into regions of positive (off) and negative (on) voltages.

correspond to the respective regions of high (negative pulse) and low (positive voltage) $W$ input powers. The off phase lasted for $2.096 \mu \mathrm{s}$, which defined a duty cycle of $80 \%$. The sum of the on and off times yields the pulse period, which was $10 \mu$ s. Deposition was also carried out using a rf signal to power the cathode. For this, a rf power supply was connected to the cathode via a matching network. The power levels led to $U_{\mathrm{sb}}$ on the substrate holder of between -200 and $-800 \mathrm{~V}$.

\section{B. Data acquisition}

A Langmuir probe (SmartProbe, Scientific Systems) was used to determine the $I-V$ characteristics of the $\mathrm{rf}$ and pulsed-dc discharges. The measuring system consisted of a circuit box connected to a data acquisition card and to an electrostatic probe. The probe consisted of a tungsten wire of $10 \mathrm{~mm}$ length with a $0.13 \mathrm{~mm}$ radius, which was placed approximately in the middle of the interelectrode gap. It thereby registered the ion and electron currents in the bulk region of the discharge. Each $I-V$ scan was the average of many $I-V$ sweeps with $1-1000$ measurements per point performed in order to increase the signal-to-noise ratio. The plasma parameters (electron temperature $T_{e}$, ion density $n_{i}$, electron density $n_{e}$, floating potential $V_{f}$, and $V_{p}$ ) were extracted from each $I-V$ curve using the model described in Sec. III.

This system setup was modified in order to monitor the plasma parameters of the pulsed-dc discharges in a timeresolved mode. ${ }^{36}$ The internal trigger of the probe box was not synchronized to $100 \mathrm{kHz}$ of the pulsed-dc signal, and the maximal measuring frequency was $\sim 5 \mathrm{kHz}$. Therefore, the Langmuir probe was connected to a time-delay circuit to perform synchronous measurements of the $I-V$ characteristics. As shown in Fig. 2, we used the external trigger entrance to the probe box for this purpose. The light signal supplied by the power source was guided through an optical fiber to a pulse conditioner, which was constituted of a photodiode followed by a signal amplifier together with an analog-digital converter. After this, the signal frequency was reduced by means of a frequency divider, and the resulting signal triggered $I-V$ acquisition. The measuring time within 


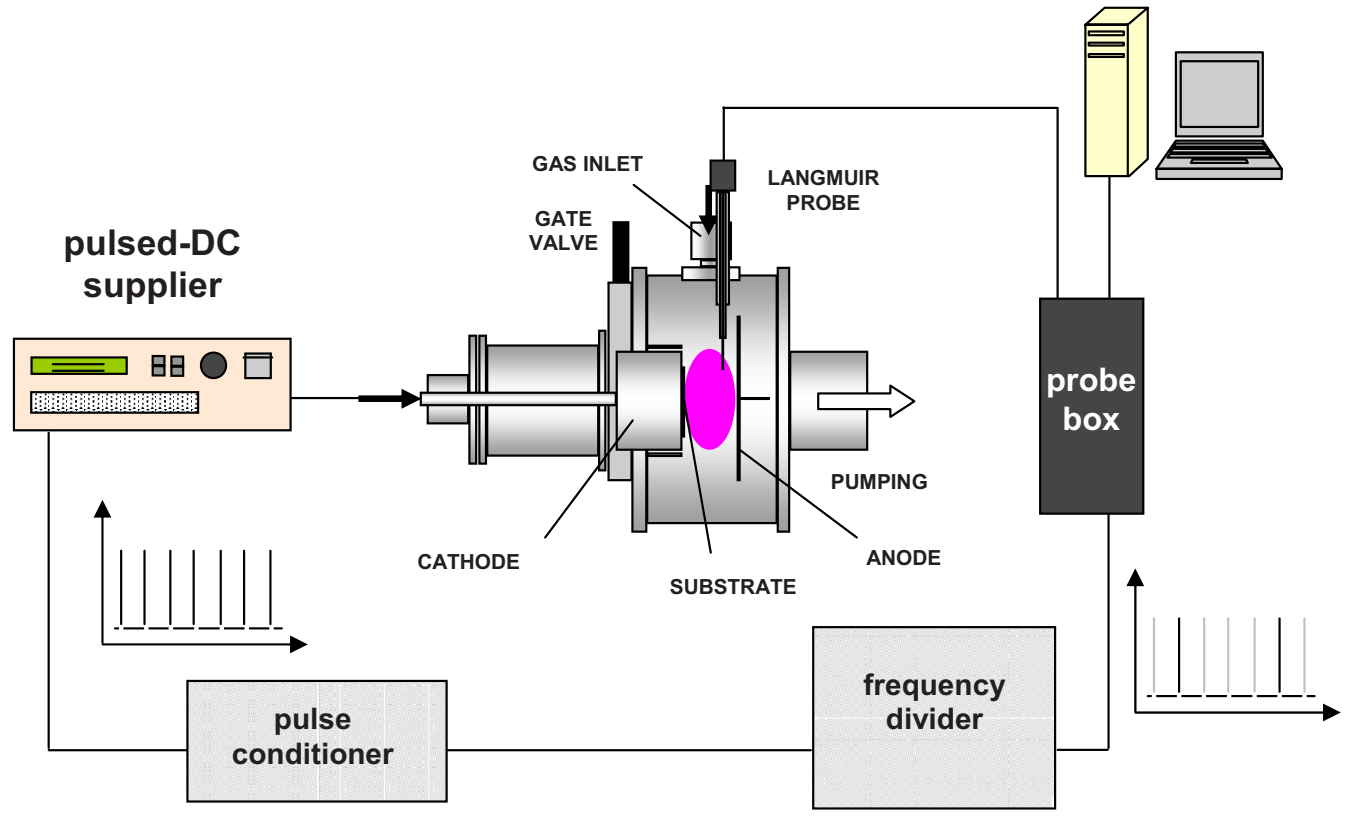

FIG. 2. (Color online) PECVD reactor where the DLC films were grown with a time-delay circuit to operate a Langmuir probe in a time-resolved mode.

the pulse was selected using a pulse generator with a timedelay actuator, which provided a time resolution of $1 \mu$ s: ten measurements per pulse.

Since the Langmuir probe was exposed to a $\mathrm{CH}_{4}$ discharge, it was at risk of becoming progressively coated by a hydrocarbon film which would distort $I-V$ acquisition. A cleaning protocol was therefore enacted prior to each measurement. This procedure consisted of biasing the probe to different $\mathrm{rf}$ voltages in Ar plasma in order to heat up the probe and to increase the ion bombardment. A discharge in oxygen $\left(\mathrm{O}_{2}\right)$ at $0.6 \mathrm{~Pa}$ was also systematically carried out to remove contaminants from the chamber walls. Prior to the $\mathrm{O}_{2}$ treatment, the probe was retracted and protected to prevent oxidation.

\section{RESULTS AND DISCUSSION}

\section{A. Analysis of the $I-V$ characteristics}

\section{Modeling}

The $I-V$ curves recorded by the Langmuir probe were modeled to extract the plasma parameters of the $\mathrm{rf}$ and pulsed-dc discharges in $\mathrm{CH}_{4}$. Assuming a nondrifting Maxwellian distribution of electrons, the current collected by the probe $i$ can be modeled as a function of the probe bias $V,{ }^{44}$

$$
i=C \sqrt{V_{P}-V}+i_{P} \exp \left[\frac{e\left(V-V_{P}\right)}{k T_{e}}\right], \quad V<V_{p},
$$

where $C$ is a constant, $e$ is the elementary charge (1.6 $\left.\times 10^{-19} \mathrm{C}\right)$, and $\kappa$ is Boltzmann's constant (1.38 $\left.\times 10^{-23} \mathrm{~J} / \mathrm{K}\right)$. The prefactor $i_{p}$ is the electron current at $V_{p}$

$$
i_{p}=n_{e} e A\left(\frac{\kappa T_{e}}{2 \pi m}\right)^{1 / 2}
$$

where $A$ is the probe area, and $m$ is the electron mass (9.1 $\times 10^{-31} \mathrm{~kg}$ ). This model is valid up to $V_{p}$ : the electron saturation region is therefore beyond the range of this model.
The first term of Eq. (1) dominates in the ion saturation region, whereas the second term accounts for the electron current in the retardation zone. The proportionality of the ion current to $\sqrt{V_{p}-V}$, which agrees well with our measurements, is based on the orbital motion limited (OML) model. The theory of OML according to Langmuir provides acceptable estimations of $n_{i}$ for weakly ionized (low density, low $p$ ) plasmas so long as the sheath is thick compared to the probe radius $r_{p} .{ }^{45}$ According to the criterion used by Chen, ${ }^{46}$ discharges fulfilling $r_{p} / \lambda_{D}<3$ may be treated using the OML formalism, where $\lambda_{D}$ is the Debye length. This parameter is related to the scale of charge fluctuations in the plasma, thus

$$
\lambda_{D}=\left(\frac{\varepsilon_{0} \kappa T_{e}}{n e^{2}}\right)^{1 / 2},
$$

where $\varepsilon_{0}$ is the permittivity of free space (8.85 $\left.\times 10^{-12} \mathrm{~F} / \mathrm{m}\right)$ and $n$ is the plasma density. The estimated values of $\lambda_{D}$ for $\mathrm{rf}$ and pulsed-de discharges fulfilled the relation with $r_{p}$ given above, thus justifying the use of OML theory to fit the $I$ - $V$ curves.

The average ion mass $M_{i}$ is dominated by the most abundant ion, which is straightforward in the case of a plasma of an inert element like Ar. However, discharges in hydrocarbon induce many different chemical processes, which produce a large variety of intermediate precursors accompanied by different ionic species. The $\mathrm{CH}_{4}$ glow discharges studied here are electropositive. According to previous analyses with QMS, $\mathrm{CH}_{4}^{+}, \mathrm{CH}_{3}^{+}, \mathrm{C}_{2} \mathrm{H}_{5}^{+}$, and $\mathrm{CH}_{5}^{+}$are the predominant ions in $\mathrm{CH}_{4}$ glow discharges: ${ }^{31,47}$ they are products of electron impact dissociation and ionization processes. In this work, we assumed that $\mathrm{CH}_{5}^{+}$was the most abundant ion, so the hypothesis $Z=17$ ( $Z$ is the atomic number) was considered in our calculations, in contrast to other values proposed in previous works. ${ }^{36}$ 


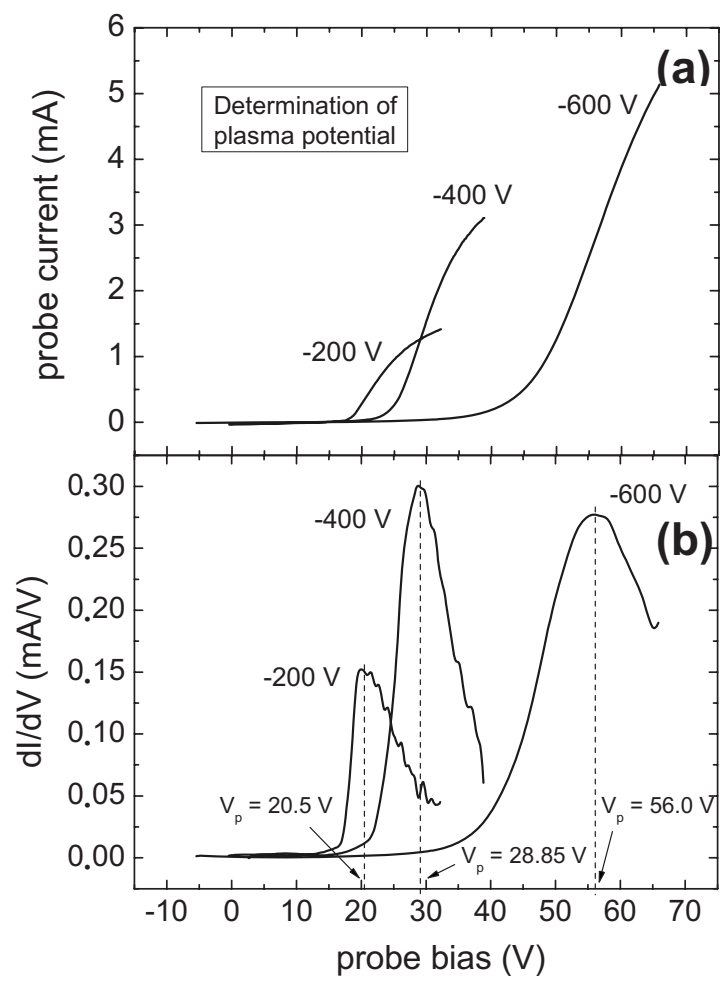

FIG. 3. (a) $I-V$ characteristics corresponding to rf discharges in $\mathrm{CH}_{4}$ powered at different $U_{\mathrm{sb}}$ and (b) their first derivatives.

\section{Calculation of the plasma parameters}

The $I-V$ curves were fitted to the above model in order to extract the plasma parameters. The procedure involved the following steps:

(i) Determination of $V_{p}$ by locating the maximum of the first derivative of $I-V$.

(ii) Plotting the $I-V$ curve with current on a logarithmic scale. In this way, the fitting of the ion saturation region is more precise.

(iii) Fitting of Eq. (1) to the logarithmic curve from (ii) above using the least-squares method and optimizing $C, n_{e}$, and $T_{e}$; a small term $I_{0}$ was added to account for small parasitic currents in the probe.

(iv) Estimation of $n_{i}$, which must equal to $n_{e}$ in order to preserve the electric neutrality of the plasma.

\section{B. rf discharges monitored by a Langmuir probe}

\section{I-V characteristics}

The $I-V$ curves resulting from rf discharges and their first derivatives corresponding to $U_{\mathrm{sb}}$ of $-200,-400$, and -600 $\mathrm{V}$ are plotted in Fig. 3. They show the common features of a
Maxwellian distribution of electrons. Namely, Eq. (1) fits the regions of ion saturation and electron retardation. The maximum of the first derivative provided $V_{p}$ at each $U_{\mathrm{sb}}$.

The $-600 \mathrm{~V}$ self-bias curve differs considerably from those for -200 and $-400 \mathrm{~V}$. The reason of this is explained in the discussion of the plasma parameters below. The $I-V$ curves have elevated signal-to-noise ratios, indicative of stable discharges.

\section{Plasma parameters}

Table I lists the plasma parameters extracted from the $I-V$ curves for the rf discharges. Each $U_{\mathrm{sb}}$ corresponded to a specific $W$, which is also given in the table. The succession of columns shows the evolution of the plasma parameters as a consequence of the $W$ variation.

$T_{e}, n_{e}$ and $V_{p}$ increased as $U_{\mathrm{sb}}$ increased. At -200 and $-400 \mathrm{~V}$ of $U_{\mathrm{sb}}, T_{e}$ was of the order of $1 \mathrm{eV}$, whereas $n_{e}$ and $V_{p}$ ranged between $1-5 \times 10^{9} \mathrm{~cm}^{-3}$ and $20-30 \mathrm{~V}$, respectively. At $U_{\mathrm{sb}}$ of $-600 \mathrm{~V}, T_{e}$ and $V_{p}$ were much higher. This suggests a substantial change in the discharge dynamics between -400 and $-600 \mathrm{~V}$. In fact, the increment step in $U_{\mathrm{sb}}$ $(200 \mathrm{~V})$ did not correspond to the size of the step in $W$, which was around $30 \mathrm{~W}$ from -200 to $-400 \mathrm{~V}$, but more than $50 \mathrm{~W}$ to reach $-600 \mathrm{~V}$ from $-400 \mathrm{~V}$. Thus, the plasma parameters responded more linearly to the increase in absorbed power than to the $U_{\mathrm{sb}}$ variation. All the parameters were notably higher at a $U_{\mathrm{sb}}$ of $-600 \mathrm{~V}$, where $n_{e}$ reached $6.1 \times 10^{9} \mathrm{~cm}^{-3}$, indicating four times the degree of ionization at $-200 \mathrm{~V}$ of self-bias.

The approximation of $Z=17$ was tested using the formula that relates $V_{p}$ and $V_{f}$ via $T_{e}$

$$
V_{p}-V_{f}=\frac{k T_{e}}{2 e} \ln \left(\frac{2 M_{i}}{\pi m}\right) \text {. }
$$

$T_{e}$ was recalculated from this expression and was in agreement with the values in Table I to within $0.5 \mathrm{eV}$. Although the sensitivity of Eq. (4) to $M_{i}$ is poor, this result supports the selection of $\mathrm{CH}_{5}^{+}$as the most abundant ion.

In the OML theory, the prefactor $C$ in Eq. (1) is proportional to $n_{i}$. Despite the relatively good fit of our $I-V$ curves to Eq. (1), the values of $n_{i}$ are normally overestimated. Ignoring distortions introduced by $\mathrm{rf}$ powering, ${ }^{48}$ this excess in $n_{i}$ is a consequence of the charge exchange collisions produced in the probe sheath. ${ }^{49}$ Therefore, $n$ was identified with $n_{e}$. More sophisticated models of the ion current that correct such overestimation due to collisions have been applied to the diagnostics of high-density plasmas $\left(n \geq 10^{11} \mathrm{~cm}^{-3}\right) .{ }^{46,50}$

TABLE I. Variation in plasma parameters for rf source in $\mathrm{CH}_{4}$ as a function of $U_{\mathrm{sb}}$ and $W$. We have assumed $n \approx n_{e}$.

\begin{tabular}{cccccc}
\hline \hline $\begin{array}{c}U_{\mathrm{sb}} \\
(\mathrm{V})\end{array}$ & $\begin{array}{c}W \\
(\mathrm{~W})\end{array}$ & $\begin{array}{c}T_{e} \\
(\mathrm{eV})\end{array}$ & $\begin{array}{c}n \\
\left(10^{9} \mathrm{~cm}^{-3}\right)\end{array}$ & $\begin{array}{c}V_{p} \\
(\mathrm{~V})\end{array}$ & $\begin{array}{c}V_{f} \\
(\mathrm{~V})\end{array}$ \\
\hline-200 & 13 & 1.0 & 1.5 & 21 & 18 \\
-400 & 43 & 1.9 & 4.7 & 29 & 23 \\
-600 & 100 & 5.3 & 6.1 & 56 & 34 \\
\hline \hline
\end{tabular}




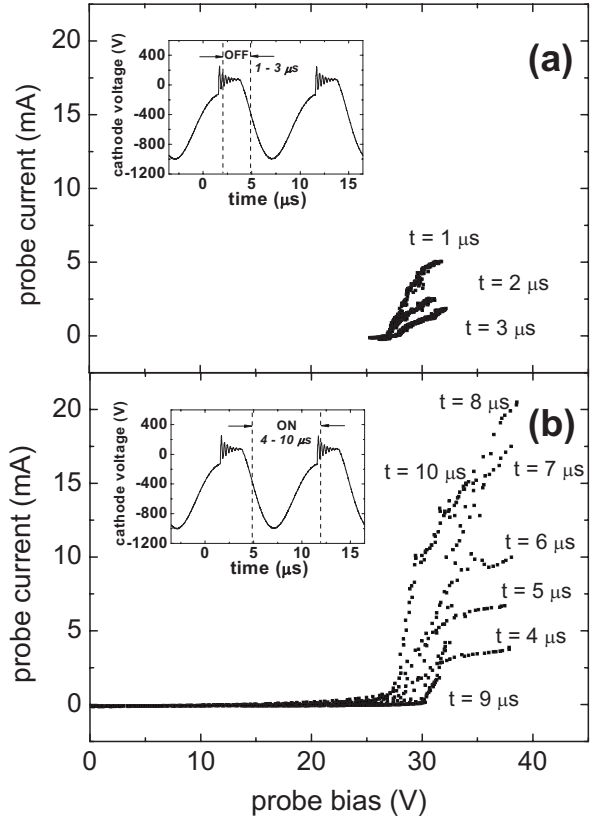

FIG. 4. $I-V$ curves for a pulsed-dc discharge in $\mathrm{CH}_{4}$ with a peak voltage of $-900 \mathrm{~V}$ recorded during the (a) off and (b) on phases. The insets show the part analyzed within the pulse cycle.

\section{Bipolar pulsed-dc discharges monitored by a fast Langmuir probe}

\section{Time-resolved recording of I-V characteristics}

Figure 4 shows the evolution with a time step of $1 \mu$ s of $I$ - $V$ curves corresponding to pulsed-dc discharges in $\mathrm{CH}_{4}$ excited by a peak voltage of $-900 \mathrm{~V}$. The curves from the off phase are displayed in Fig. 4(a). They show a progressive weakening of the probe signal due to the low positive bias of the electrode. These measurements show a low signal-tonoise ratio, which is evidence of a very weak discharge. The signals measured during the on phase were more intense [Fig. 4(b)]. Their shapes reveal the non-Maxwellian nature of the plasma.

Each characteristic was fitted to the $I-V$ model described above to obtain the plasma parameters (Fig. 5). The curves for the off region fit the model for Maxwellian electrons [Eq. (2)]. Despite the low intensity of the signal (which made fitting difficult), the plasma parameters indicated active discharge. This is confirmed by the smooth variation of $V_{p}$ during the pulse, as discussed below. However, the on phase yielded $I-V$ curves that could not be fitted to the model above. Instead, they were successfully fitted to a twotemperature model. To do this, Eq. (1) was generalized to the following [which accounts for the presence of two electron populations (cold and hot) in the on phase]:

$$
\begin{aligned}
i= & C \sqrt{V_{P}-V}+i_{p}^{\mathrm{hot}} \exp \left[\frac{e\left(V-V_{P}\right)}{k T_{e}^{\mathrm{hot}}}\right] \\
& +i_{p}^{\text {cold }} \exp \left[\frac{e\left(V-V_{P}\right)}{k T_{e}^{\text {cold }}}\right], \quad V<V_{p} .
\end{aligned}
$$

Here, the intensities at $V_{p}$ have the same dependences as in Eq. (2),
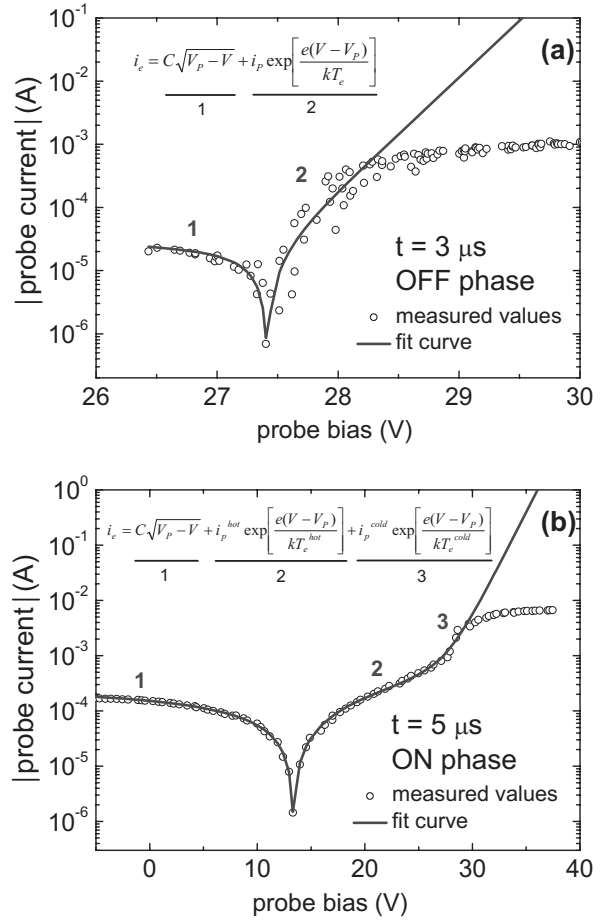

FIG. 5. Respective fittings of Eqs. (1) and (5) to $I-V$ curves registered during the (a) off and (b) on phases of a pulsed-dc discharge. The absolute values of current are plotted on a log scale.

$$
\begin{aligned}
& i_{p}^{\text {hot }}=n_{e}^{\text {hot }} e A\left(\frac{k T_{e}^{\mathrm{hot}}}{2 \pi m}\right)^{1 / 2}, \\
& i_{p}^{\text {cold }}=n_{e}^{\text {cold }} e A\left(\frac{k T_{e}^{\text {cold }}}{2 \pi m}\right)^{1 / 2} .
\end{aligned}
$$

Then, $n_{e}$ is calculated as

$$
n_{e}=n_{e}^{\text {hot }}+n_{e}^{\text {cold }} \text {. }
$$

Figure 5, which is plotted against a logarithmic scale, shows the Maxwellian electron distribution in the off phase, which switches to a bi-Maxwellian distribution as soon as the on phase of the pulse started. Naturally, these two groups of electrons merge again into a single population at the start of the next off phase.

Two-temperature electron populations are typically found in weakly ionized plasmas at low $p$ and indicate that the electron energy distribution function departs from thermodynamic equilibrium. Sheridan et al. ${ }^{51}$ reported them for dc discharges in helium and Godyak et al. ${ }^{52}$ analyzed different non-Maxwellian plasmas with probe diagnostics. In the field of pulsed plasmas, Bäcker and Bradley ${ }^{37}$ thoroughly investigated the effects of incorporating magnetrons into the plasma parameters, such as, for instance, the generation of bi-Maxwellian discharges induced by the presence of a magnetic field.

\section{Tracking plasma parameters}

a. Electron temperature Figure 6 shows the evolution of $T_{e}$ within pulses with a peak voltage of $-700,-900$, and $-1100 \mathrm{~V}$. The behavior of this variable was clearly differentiated between the regions of the pulse. During the off phase, 


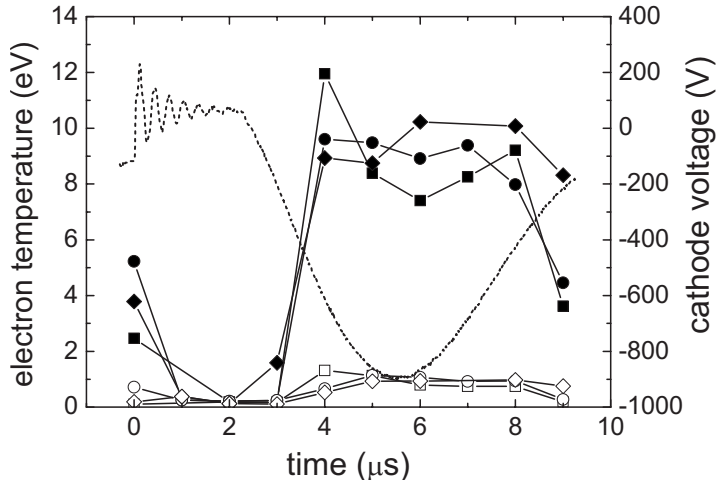

FIG. 6. Evolution of $T_{e}^{\text {cold }}$ and $T_{e}^{\text {hot }}$ together with the pulse waveform (dotted line). Solid symbols correspond to the hot population and outlines correspond to the cold group. Squares: $-700 \mathrm{~V}$; circles: $-900 \mathrm{~V}$; rhombuses: $-1100 \mathrm{~V}$.

there was a single group of electrons with a $T_{e}$ of less than 1 $\mathrm{eV}$. This was followed by the generation of a hot population of electrons within the initial $2 \mu$ s of the on phase. The hot electrons had temperatures of over $10 \mathrm{eV}$, which then fell during this phase, whereas the cold group reached a plateau of the order of $1 \mathrm{eV}$. Bradley et al..$^{53}$ observed a hot burst of electrons confined by means of a magnetron in plasma subjected to $100 \mathrm{kHz}$ dc pulses. Their $T_{e}$ peaked at transition between the on and off phases.

The splitting into two of the electron population of the pulsed-dc plasma might be the result of an additional heating mechanism that is activated during the on phase. The literature reports that the oscillating sheath edge at the cathode is a relevant source of hot electrons. ${ }^{46}$ Such excitation is known as stochastic heating and it selectively excites high-energy electrons at low $p$, while low-energy electrons remain cold. The selectivity of such energy transfer is the key to the electrons forming a bi-Maxwellian population. The on time may have been too short to avoid this burst of hot electrons cooling down and merging with the cold population. This hypothesis of thermalization should be verified by long-term analysis of the temperature decay within a pulse.

Bäcker and Bradley ${ }^{37}$ supposed that secondary electrons from the cathode were firmly pushed into the bulk of the plasma by the contracting sheath, leading to an increase in $T_{e}$. Using Langmuir probe diagnostics, Glocker showed that ac discharges resulted in electrons that were more energetic than in dc-powered plasmas. This was attributed to the rapidly advancing sheath edge at the beginning of the on phase when a burst of hot electrons coming from the sheath was injected into the plasma. ${ }^{54}$ This effect may be further explained by considering the ionic speed of sound (Bohm velocity) $u_{i}{ }^{55}$

$$
u_{i}=\left(\frac{\kappa T_{e}}{M_{i}}\right)^{1 / 2} .
$$

According to the values of $T_{e}$ and $n_{e}$ at the off-on transition, $u_{i}$ was lower than $2000 \mathrm{~m} / \mathrm{s}$, whereas the sheath-edge velocity was more than $10^{4} \mathrm{~m} / \mathrm{s}$. This velocity was estimated from the temporal variation in the collisional sheath thickness $s,{ }^{44}$

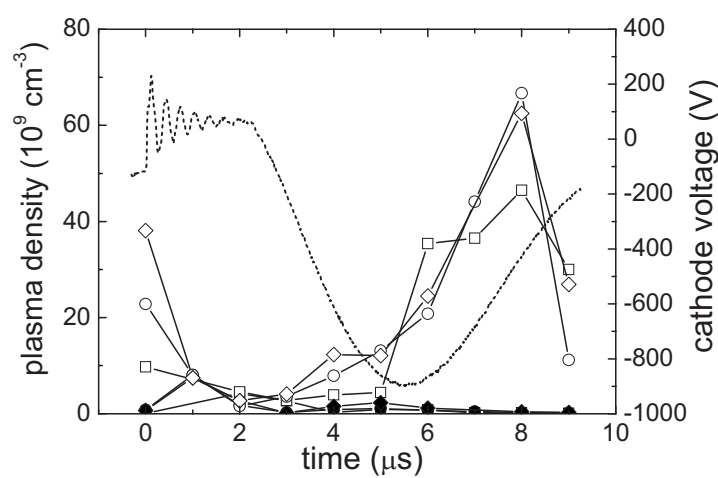

FIG. 7. Evolution of $n_{e}^{\text {hot }}$ and $n_{e}^{\text {cold }}$ together with the pulse waveform (dotted line). Solid symbols correspond to the hot electrons and outlines correspond to the cold electrons. Squares: $-700 \mathrm{~V}$; circles: $-900 \mathrm{~V}$; rhombuses: -1100 $\mathrm{V}$.

$$
s=\left[\frac{4.12}{\pi}\left(\frac{e V_{0}}{\kappa T_{e}}\right)^{3} \lambda_{i} \lambda_{D s}^{4}\right]^{1 / 5},
$$

where $\lambda_{i}$ is the mean free path of the ions and $\lambda_{D s}$ is the Debye length at the sheath edge. According to Peter et al., ${ }^{42}$ the value of $\lambda_{i}$ for $\mathrm{CH}_{4}$ at a $p$ of $10 \mathrm{~Pa}$ is around $1.2 \mathrm{~mm}$, which is of the order of or lower than $s$. The value of $n$ at the sheath edge is assumed to be $n_{s} \approx 0.63 n_{e}$. In this case, $V_{0}$ equals $V_{p}$ minus the cathode voltage.

Since the sheath edge moved faster than the ionic sound within the first microsecond of the on phase, instabilities generated by energetic acoustic waves could be associated with the burst of hot electrons. This could be the key to achieving high $n$ in pulsed-dc discharges, as explained below. This hypothesis, based on the rapid motion of ions, cannot be used for rf discharges, in which the ions constantly flow toward the reactor walls since they cannot follow the oscillations of the electric field. This, together with the fact that collisionless heating becomes inefficient at higher frequencies, ${ }^{56}$ might explain why the rf discharges in $\mathrm{CH}_{4}$ did not result in bi-Maxwellian distributions.

b. Plasma density $n_{e}$ was evaluated every $1 \mu$ s from the probe current at $V_{p}$, and its behavior during the pulse is plotted in Fig. 7. It oscillated between $2 \times 10^{9}$ and 8 $\times 10^{9} \mathrm{~cm}^{-3}$ in the off region. As opposed to $T_{e}, n_{e}^{\text {cold }}$ gradually increased once the on phase started and peaked at $\sim 2.5 \mu$ s after the peak voltage. Similar patterns were observed by Lieberman and Ashida ${ }^{57}$ and Richter et al. ${ }^{38}$ This delay in reaching the maximum can be attributed to the relatively high inertia of ions reacting to perturbations of the electric field, compared to the fast response of the electrons. The response time of each species is limited by its respective plasma frequency. After the peak, $n_{e}^{\text {cold }}$ fell sharply, coinciding with the positive voltage region.

The discharges at -900 and $-1100 \mathrm{~V}$ produced an exponential evolution in $n_{e}^{\text {cold }}$ until it reached its maximal value, which surpassed $6 \times 10^{10} \mathrm{~cm}^{-3}: 10$ times higher than in the case of the rf signal. A smaller increase was measured for less energetic pulses $(-700 \mathrm{~V})$, where $n_{e}^{\text {cold }}$ approached $5 \times 10^{10} \mathrm{~cm}^{-3}$. The hot electrons had a relatively low density all the time: generally below $10^{9} \mathrm{~cm}^{-3}$. This may represent the reduced fraction of hot electrons boosted by the sheath edge when the on phase started. As the pulse continued, the 


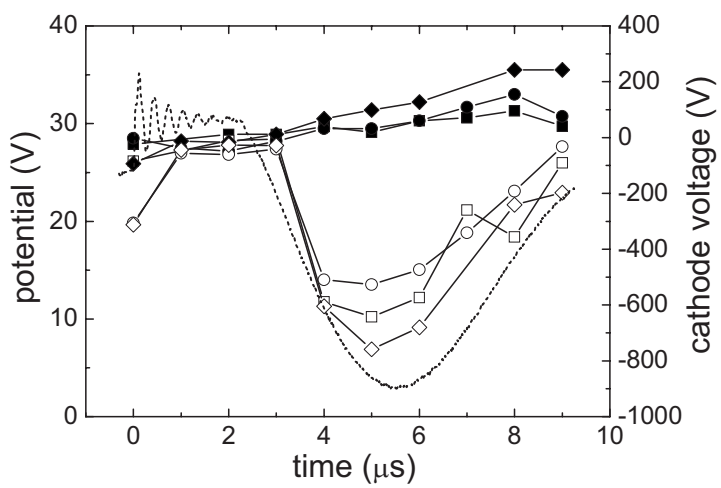

FIG. 8. Evolution of $V_{p}$ and $V_{f}$ together with the pulse waveform (dotted line). Solid symbols correspond to $V_{p}$ and outlines correspond to $V_{f}$. Squares: $-700 \mathrm{~V}$; circles: $-900 \mathrm{~V}$; rhombuses: $-1100 \mathrm{~V}$.

cold population increased, corresponding to a decrease in $n_{e}^{\text {hot }}$ and in $T_{e}^{\text {hot }}$. This indicates the cooling down of the hot group, whose energy was partly spent enhancing the ionization.

Due to the strong oscillations of the probe sheath associated with pulsed plasmas, time-resolved measurements of $n_{i}$ were not reliable. ${ }^{58}$ This is especially true for the off phase when the probe sheath expands so much that $n_{i}$ is overestimated according to Ref. 45. The low signal-to-noise ratio of the recorded $I-V$ curves was another problem during the off phase. Therefore, the corresponding data points were not considered. However, the velocity of the ion response to voltage changes was limited by the ion plasma frequency, as indicated above. Electrons are the best tracers of fast transitions in the driving voltage and $V_{p}$. The value of $n$ is therefore more accurately monitored by considering $n_{e}$, not $n_{i}$ calculated for the ion saturation region.

c. Plasma and floating potentials Figure 8 shows the evolution of $V_{p}$ and $V_{f}$ during the pulse. $V_{p}$, which ranged from 25 to $35 \mathrm{~V}$, showed a monotonic and smooth increase during the whole period. It only decreased sharply at the transition from on to off when the polarity of the electrode was inverted. The fact that $V_{p}$ did not vanish during the off phase indicates that the discharge was never completely extinguished. This is an important point with regard to plasma stability, the minimization of arcs, and maintaining the deposition rate. Since $V_{p}$ was stable during the off phase, we can infer that the decay time of the plasma was longer than $2 \mu \mathrm{s}$. This is related to the time scale of charge redistribution and recombination in the positive voltage region, which is limited by the ion motion.

$V_{f}$ evolved parallel to the cathode voltage: its behavior was connected to the variation in $T_{e}$ according to Eq. (5). From this expression an "effective" $T_{e}$ can be derived, whose value is between $T_{e}^{\text {hot }}$ and $T_{e}^{\text {cold }}$.

\section{Averaged parameters}

Table II gives the plasma parameters averaged over one period for different peak voltages. $T_{e}^{\text {hot }}$ and $T_{e}^{\text {cold }}$ show no definite trend with respect to $W$. Increasing $W$ did influence $n_{e}$, although variation was small. The same applies to $V_{p}$ and $V_{f}$. In contrast to the $U_{\mathrm{sb}}$ resulting from rf discharges, the variation in the peak voltage in pulsed-dc $\mathrm{CH}_{4}$ discharges did not produce major changes in the plasma parameters. Thus, the peak voltage as well as $W$ can be used as external parameters to control the ion energy without drastically changing the discharge structure.

$n$ was treated as the estimated value of $n_{e}$. As noted above, evaluations of $n$ from the ion saturation region in pulsed plasmas are imprecise and lead to important deviations from electrical neutrality.

It is worth noting that the average $n$ surpassed 2 $\times 10^{10} \mathrm{~cm}^{-3}$, which correspond to ionization rates between three and four times higher than for $\mathrm{rf}$ discharges. Therefore, pulsed-dc power not only provided large ion energies through high peak voltages, but it also gave rise to more active plasmas, thanks to stronger ionizations than those produced by the rf source. This could explain the significant increase in DLC deposition rates obtained using pulsed-dc compared to rf PECVD, as reported by Andújar et al. ${ }^{24}$ However, DLC deposition is associated with the on phase, where the plasma becomes more active. During the off phase, ionization was weaker as indicated by the lower values of $T_{e}$ and $n_{e}$. This leads to a reduction in compressive stress due to the accommodation of the deposited species at more stable sites. $^{24}$

\section{Input power and deposition rate \\ 1. Growth regimes}

We analyzed the growth of the DLC films through the technical parameters of deposition. We studied the dependence of the deposition rate on $W$ into the $\mathrm{CH}_{4}$ discharge. $W$ per unit gas flow $W / F$ is a parameter that is useful for studying the dynamics of PECVD from hydrocarbon precursors. ${ }^{14,59} \mathrm{~W} / \mathrm{F}$ can be interpreted as the rate of energy imparted per particle, and it is related to the mass deposition rate $R_{m}$ through the following expression, which is valid for radical-dominated plasma processes at moderate energies: ${ }^{60,61}$

$$
\frac{R_{m}}{F}=G \exp \left(-\frac{E_{a}}{W / F}\right),
$$

where $F$ is the gas flow and $G$ is a reactor-dependent factor. The parameter $E_{a}$ is a characteristic of the gas used, and it is

TABLE II. Plasma parameters averaged over one pulse for $100 \mathrm{kHz}$ pulsed-dc discharges in $\mathrm{CH}_{4}$ at different $\mathrm{W}$. We have assumed $n \approx n_{e}^{\text {cold }}+n_{e}^{\text {hot }}$.

\begin{tabular}{ccccccccc}
\hline \hline $\begin{array}{c}\text { Peak voltage } \\
(\mathrm{V})\end{array}$ & $\begin{array}{c}W \\
(\mathrm{~W})\end{array}$ & $\begin{array}{c}T_{e}^{\text {hot }} \\
(\mathrm{eV})\end{array}$ & $\begin{array}{c}T_{e}^{\text {cold }} \\
(\mathrm{eV})\end{array}$ & $\begin{array}{c}n_{e}^{\text {hot }} \\
\left(10^{9} \mathrm{~cm}^{-3}\right)\end{array}$ & $\begin{array}{c}n_{e}^{\text {cold }} \\
\left(10^{9} \mathrm{~cm}^{-3}\right)\end{array}$ & $\begin{array}{c}n \\
\left(10^{9} \mathrm{~cm}^{-3}\right)\end{array}$ & $\begin{array}{c}V_{p} \\
(\mathrm{~V})\end{array}$ & $\begin{array}{c}V_{f} \\
(\mathrm{~V})\end{array}$ \\
\hline-700 & 18 & 5.7 & 0.60 & 1.0 & 19 & 20 & 30 & 20 \\
-900 & 34 & 5.6 & 0.64 & 1.4 & 20 & 21 & 30 & 21 \\
-1100 & 52 & 5.8 & 0.55 & 1.9 & 21 & 23 & 31 & 19 \\
\hline \hline
\end{tabular}




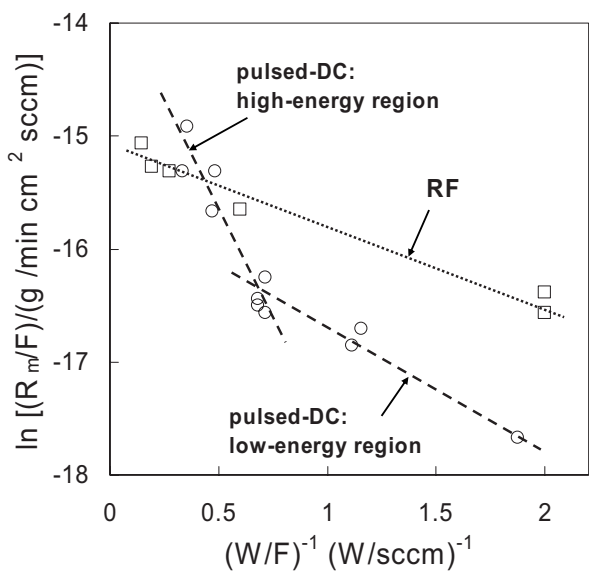

FIG. 9. Arrhenius-type plots that relate $R_{m}$ to $W$ for DLC samples deposited by rf and pulsed-dc discharges in $\mathrm{CH}_{4}$.

defined as the energy required to yielding significant fragmentation and polymerization of the precursor. The factor $G$ is proportional to the maximal $x_{\text {dep }}$ of the precursor gas to deposited film, which indicates the deposition efficiency ${ }^{62}$

$$
x_{\mathrm{dep}}=G A_{\mathrm{dep}} \frac{R T_{0}}{M_{p} p_{0}} .
$$

In this formula, $A_{\text {dep }}$ is the deposition area, $R$ is the ideal gas constant $\left(8.31 \mathrm{~J} \mathrm{~K}^{-1} \mathrm{~mol}^{-1}\right), M_{p}$ is the molecular mass of the precursor, and $T_{0}$ and $p_{0}$ are the respective temperature and pressure under standard conditions. The average density of DLC is considered to be $1.9 \mathrm{~g} / \mathrm{cm}^{3} .{ }^{36}$ Equation (11) requires that the residence time of gas species $\tau$ is much longer than the pulse period. ${ }^{30}$ This condition is fulfilled since $\tau$ is $2.8 \mathrm{~s}$. Hegemann et al. ${ }^{61,62}$ used this formalism to study polymer depositions by PECVD. Bauer et al. ${ }^{63}$ considered the average energy dissipated per gas molecule in a pulsed discharge, a parameter similar to $W / F$, which permitted the control of the plasma chemistry.

Figure 9 shows the Arrhenius-type plots of $R_{m} / F$ as a function of $(W / F)^{-1}$ for DLC depositions and Table III lists the parameters fitted from Eq. (11). The range of $W / F$ analyzed is within the optimal interval for film deposition from $\mathrm{CH}_{4}$ plasma, as reported in Ref. 28. The data points for the rf processes are linear. $E_{a}$ and $G$ were extracted from the linear fit analysis of these data using Eq. (11). A value of $41.4 \mathrm{~J} / \mathrm{cm}^{3}$ was obtained for $E_{a}$, in agreement with that reported by Hegemann ${ }^{64}$ for an asymmetric rf discharge in $\mathrm{CH}_{4}$. The low $x_{\text {dep }}$ of only $2.9 \%$ indicates a process with poor conversion of the precursor. In the plot of growth processes using pulsed-dc energy, two regions with different $E_{a}$ were

TABLE III. Parameters from Eqs. (11) and (12) for the PECVD of DLC films grown with $\mathrm{rf}$ and pulsed-dc energy.

\begin{tabular}{lccc}
\hline \hline & $\begin{array}{c}E_{a} \\
\left(\mathrm{~J} \mathrm{~cm}^{-3}\right)\end{array}$ & $\left.\begin{array}{c}G \\
\left(\times 10^{-7}\right.\end{array} \mathrm{g} \mathrm{min}^{-1} \mathrm{~cm}^{-2} \mathrm{SCCM}^{-1}\right)$ & $\begin{array}{c}X_{\text {dep }} \\
(\%)\end{array}$ \\
\hline $\mathrm{rf}$ & 41.4 & 2.8 & 2.9 \\
Pulsed-dc (low $E)$ & 60.6 & 1.5 & 1.6 \\
Pulsed-dc (high $E)$ & 228 & 10 & 11 \\
\hline \hline
\end{tabular}

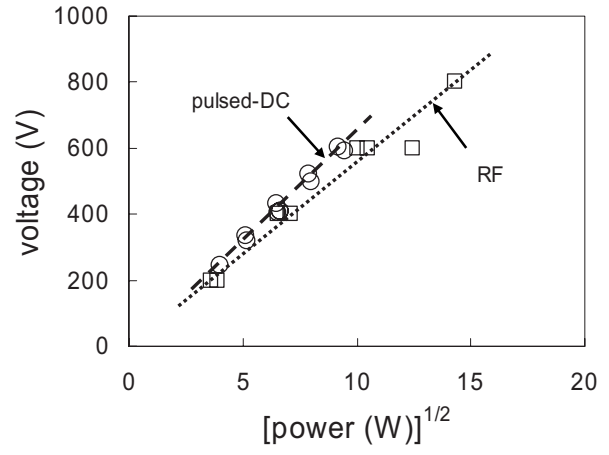

FIG. 10. Variation in the cathode voltage with $W$ for rf- and pulsed-DC PECVD processes.

detected. The value of $W / F$ corresponding to a peak voltage of $-1000 \mathrm{~V}$ marked the limit between low- and high-energy processes.

The low-energy region is located on the right of the Arrhenius-type plot. It is associated with a set of growth mechanisms, which are characterized by an $E_{a}$ of $60.6 \mathrm{~J} / \mathrm{cm}^{3}$ and an $x_{\text {dep }}$ of $1.6 \%$. As $W$ increased, the system crossed the energy threshold and entered the high-energy region. There, a second set of growth mechanisms dominated DLC deposition. This regime was more efficient than that which was dominant at low energies with $x_{\text {dep }}$ reaching $11 \%$. Moreover, $E_{a}$ increased by a factor of 4 , indicating that the deposition process involved increased decomposition of the $\mathrm{CH}_{4}$ molecules at high electric fields. $E_{a}$ is connected to the bond breakage energy of the precursor molecule. ${ }^{61}$ Therefore, the change in $E_{a}$ accounts for the activation of new intermediate precursors responsible for film deposition. Finally, rf and low-energy pulsed-dc discharges of $\mathrm{CH}_{4}$ presented similar values of $E_{a}$. This suggests comparable plasma chemistry and kinetics in the two types of excitation.

\section{Power dissipation modes}

The heating mechanism dominant in a glow discharge depends strongly on $p$. According to Lieberman and Lichtenberg, ${ }^{44}$ our discharges were cold plasmas developed between intermediate- and high- $p$ regimes because $\lambda_{i} / d$ $\approx T_{i} / T_{e}$, where $d$ is the interelectrode distance. The ion temperature $T_{i}$ was close to room temperature. The dominant heating mechanism for of discharges in $\mathrm{CH}_{4}$ at this $p$ was observed by Peter et al. ${ }^{42}$ to be Ohmic heating. Processes heated by such a mechanism fulfill

$$
\frac{-U_{\mathrm{sb}}}{W^{1 / 2}}=A-B p,
$$

where $A$ and $B$ are empirical constants. Figure 10 shows that our rf discharges followed an Ohmic regime since $U_{\mathrm{sb}}$ $\sim W^{1 / 2}$. Pulsed-dc plasmas were also analyzed using this formalism, where $U_{\mathrm{sb}}$ was substituted by the average pulse voltage. From Fig. 10 one could infer that Ohmic heating was also dominant for the pulsed-dc discharges, although stochastic heating played an important role at the pulse time scales, as discussed in Sec. III C 2.

Figure 10 suggests that the associated resistance of the pulsed-dc plasma was independent of $W$, despite the changes 


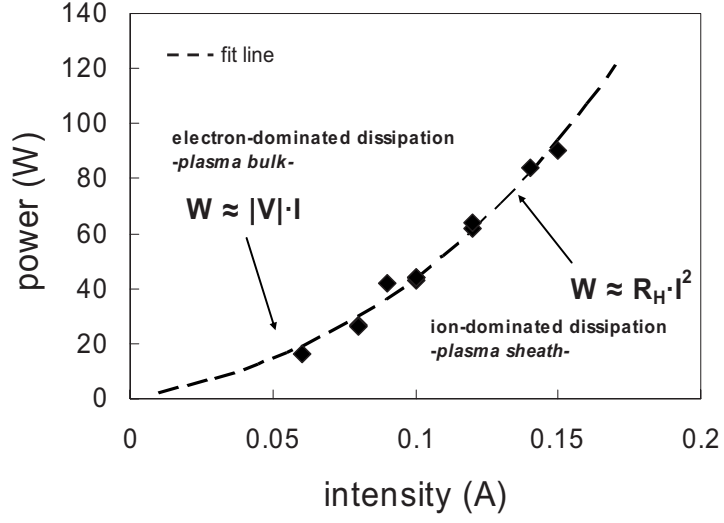

FIG. 11. Evolution of $W$ with $I$ measured for pulsed-dc plasmas in $\mathrm{CH}_{4}$.

in the growth regime and $E_{a}$ observed in Fig. 9. The analysis of the power dissipation mode in the discharge may shed some light on this issue. It is accepted that the power share in rf discharges depends on the driving intensity $I$ according to ${ }^{65}$

$$
W_{\text {total }}=W_{\text {bulk }}+W_{\text {sheath }}=|V| I+R_{\text {sh }} I^{2},
$$

where $W_{\text {total }}$ is the total power absorbed by the plasma, $W_{\text {bulk }}$ and $W_{\text {sheath }}$ are the power consumed by the bulk of the plasma and the sheath, respectively, $|V|$ is an effective plasma potential, and $R_{\mathrm{sh}}$ is the sheath resistance: $R_{\mathrm{sh}} \sim I^{0.5}$. Many experimental studies also confirm the dependence of $R_{\text {sh }}$ on the frequency and pressure. ${ }^{66-68}$ At low $W$, the energy is preferably deposited in the plasma bulk, but as $W$ increases the dissipation zone is displaced to the sheath. Figure 11 shows this evolution for pulsed-dc discharges.

Figure 12 maps the evolution of the power share as a function of the parameter $W / F$ by fitting Eq. (14) to the data from pulsed-dc $\mathrm{CH}_{4}$ discharges. The relation $W_{\text {sheath }} / W_{\text {total }}$ evolves parallel to $R_{m} / F$ versus $(W / F)^{-1}$ displayed in Fig. 9. Moreover, the transition of the power dissipation mode coincides with the threshold power for the transition of growth regimes.

The depositions performed in the low-energy region (on the right in Fig. 9) corresponded to processes in which power dissipation was bulk dominated. There, the energy supplied was mainly consumed by the electrons populating the bulk.

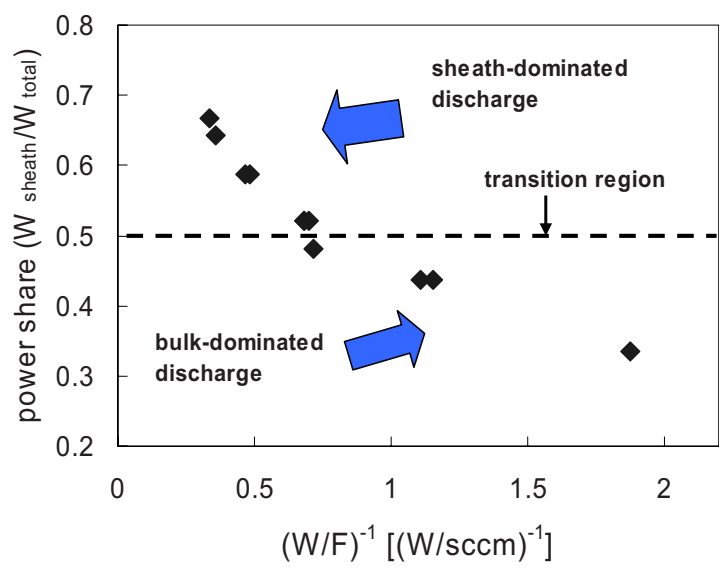

FIG. 12. (Color online) Power share in plasma volume as a function of $(W / F)^{-1}$.
However, the high-energy processes (on the left in Fig. 9) were sheath dominated, i.e., the ions in the plasma sheath absorbed the highest fraction of $W$. First, we can conclude that low-energy pulsed-dc discharges provoked a little dissociation of the $\mathrm{CH}_{4}$ molecules and the majority of $W$ was transferred to the chemical processes taking place in the bulk of the plasma. This poor decomposition of $\mathrm{CH}_{4}$ is reflected by the relatively low $E_{a}$. Second, the high-energy processes were characterized by an increased degree of dissociation of the precursor, which corresponds to a displacement of power dissipation to the sheath. This could be explained by energy saturation of the electrons in the plasma bulk. The excess energy is then utilized to accelerate the ions in the sheath toward the cathode and to enhance the stochastic heating of the electrons.

\section{CONCLUSIONS}

The study of bipolar pulsed-dc $\mathrm{CH}_{4}$ discharges to deposit DLC films was successfully performed by means of plasma diagnostics with a fast Langmuir probe. The time-resolved monitoring of the plasma parameters showed many peculiarities of this type of plasmas, as compared to rf discharges. Dividing the pulses into on and off phases provided a practical way to discuss parameter evolution within a cycle. From this analysis, we observed that the pulsed-dc discharges did not bring about a stationary state. The almost constant $V_{p}$ was indicative of a never-vanishing discharge. $T_{e}$ and $n_{e}$ showed relatively low values during the off phase. Electrons at two different temperatures were detected during the on phase, probably due to stochastic heating from the rapidly advancing sheath edge during the polarity reversal. Values of around $10 \mathrm{eV}$ were measured for the hot electrons, which had a low-density population. The cold electrons, which reached almost $7 \times 10^{10} \mathrm{~cm}^{-3}$ at the end of the on phase, constituted the majority population. The temporal evolution of the plasma parameters, together with the energetic ions generated by the peak voltage, was advantageous for the growth of DLC films with improved mechanical properties. Moreover, the plasma parameters showed a relatively smooth response to the variation in the peak voltage. Other distinctive points of pulsed-dc plasmas were the two different growth regimes, as determined by the parameter $W / F$, due to the variation in $E_{a}$. The high-energy depositions had higher $x_{\mathrm{dep}}$ and caused more dissociations of the precursor molecules. This behavior was connected to a transition from bulk- to sheath-dominated power dissipation. Table IV summarizes the results of the comparative study.

This work has shown fundamental strengths of using pulsed-dc plasmas to deposit DLC films from $\mathrm{CH}_{4}$ atmospheres. A more complete study requires the exploration of the remaining pulse variables (duty cycle, frequency, etc.) as well as the evaluation of the plasma parameters in spaceresolved mode and with alternative techniques.

\section{ACKNOWLEDGMENTS}

This research was partially supported by the projects MAT2003-02997 and DPI2007-61349 of the Spanish Ministry of Education and Science. C.C. and M.R. acknowledge 
TABLE IV. Main characteristics of rf and pulsed-de discharges in $\mathrm{CH}_{4}$ used to grow DLC films.

\begin{tabular}{|c|c|}
\hline $\mathrm{rf}(13.56 \mathrm{MHz})$ & Pulsed-dc $(100$ kHz $)$ \\
\hline One input parameter $\left(U_{\mathrm{sb}}\right)$ & Tailoring of signal (peak voltage, duty cycle, positive voltage) \\
\hline Discharge in steady state & Succession of transient states \\
\hline Maximal ion energy around $500 \mathrm{eV}$ & Peak voltage up to $-1400 \mathrm{~V}$ \\
\hline Plasma parameters very sensitive to $U_{\mathrm{sb}}$ & Smooth variation in plasma parameters with peak voltage \\
\hline One $T_{e}$ (Maxwellian population) & $T_{e}^{\text {hot }}$ and $T_{e}^{\text {cold }}($ bi-Maxwellian population) \\
\hline $10^{-5}$ degree of ionization at $-600 \mathrm{~V}$ self-bias & Peak in degree of ionization ten times higher than for $\mathrm{rf}$ \\
\hline Low $x_{\mathrm{dep}}$ & Greater deposition efficiency \\
\hline $\begin{array}{l}\text { Plasma processes described by a constant } E_{a} \\
\text { Ohmic heating }\end{array}$ & $\begin{array}{l}\text { Two growth regimes indicated by a variation in } E_{a} \\
\qquad \text { Ohmic + stochastic heating }\end{array}$ \\
\hline
\end{tabular}

the financial support from the Spanish Ministry of Science and Innovation (Juan de la Cierva contract) and from the Spanish Research Council (Grant No. UAC-2005-0021), respectively.

${ }^{1}$ T. Lampe, S. Eisenberg, and E. Rodríguez Cabeo, Surf. Coat. Technol. 174-175, 1 (2003).

${ }^{2}$ R. Hauert and J. Patscheider, Adv. Eng. Mater. 2, 247 (2000).

${ }^{3}$ M. Ohring, The Materials Science of Thin Films (Academic, New York, 2002).

${ }^{4}$ J. R. Roth, Industrial Plasma Engineering (Institute of Physics, University of Reading, Berkshire, 2001), Vol. 2.

${ }^{5}$ R. A. Scholl, Surf. Coat. Technol. 98, 823 (1998).

${ }^{6}$ J. Laimer, M. Fink, T. A. Beer, and H. Störi, Surf. Coat. Technol. 174-175, 118 (2003).

${ }^{7}$ A. Lacoste and J. Pelletier, in Plasma Polymers and Related Materials, edited by M. Mutlu, G. Dinescu, R. Förch, J. M. Martín-Martínez, and J. Vyscocyl (Hacettepe University Press, Ankara, 2005), p. 1.

${ }^{8}$ M. Liehr, S. Wieder, and M. Dieguez-Campo, Thin Solid Films 502, 9 (2006).

${ }^{9}$ H. Yasuda, Luminous Chemical Vapor Deposition and Interface Engineering (Marcel Dekker, New York, 2005).

${ }^{10}$ H.-U. Poll and S. Schreiter, Surf. Coat. Technol. 93, 105 (1997).

${ }^{11}$ J. Schmitt, M. Elyaakoubi, and L. Sansonnens, Plasma Sources Sci. Technol. 11, A206 (2002)

${ }^{12}$ J. Perrin, J. Schmitt, C. Hollenstein, A. Howling, and L. Sansonnens, Plasma Phys. Controlled Fusion 42, B353 (2000).

${ }^{13} \mathrm{~N}$. Kaiser and H. K. Pulker, Optical Interference Coatings (Springer, Berlin, 2003).

${ }^{14}$ C. Corbella, I. Bialuch, M. Kleinschmidt, and K. Bewilogua, Solid State Sci., in press (2009).

${ }^{15}$ S. Röhlecke, R. Tews, A. Kottwitz, and K. Schade, Surf. Coat. Technol 74-75, 259 (1995)

${ }^{16}$ E. H. A. Dekempeneer, L. Poirier, J. P. Lebrun, A. Pasgrimaud, Y. Desalos, and F. Balanck, Surf. Coat. Technol. 151-152, 462 (2002).

${ }^{17}$ T. Michler, M. Grischke, I. Traus, K. Bewilogua, and H. Dimigen, Diamond Relat. Mater. 7, 459 (1998).

${ }^{18}$ A. Anders, Surf. Coat. Technol. 200, 1893 (2005).

${ }^{19}$ C. Corbella, I. Bialuch, M. Kleinschmidt, and K. Bewilogua, Thin Solid Films 517, 1125 (2008).

${ }^{20}$ S. Aisenberg and R. Chabot, J. Appl. Phys. 42, 2953 (1971).

${ }^{21}$ J. Robertson, Mater. Sci. Eng. R. 37, 129 (2002).

${ }^{22}$ R. Hauert, Tribol. Int. 37, 991 (2004).

${ }^{23} \mathrm{C}$. Donnet and A. Erdemir, Tribology of Diamond-Like Carbon Films. Fundamentals and Applications (Springer, New York, 2008).

${ }^{24}$ J. L. Andújar, M. Vives, C. Corbella, and E. Bertran, Diamond Relat. Mater. 12, 98 (2003).

${ }^{25}$ A. von Keudell, M. Meier, and C. Hopf, Diamond Relat. Mater. 11, 969 (2002).

${ }^{26}$ C. Corbella, B. Echebarría, L. Ramírez-Piscina, E. Pascual, J. L. Andújar, and E. Bertran, Appl. Phys. Lett. 87, 213117 (2005).

${ }^{27}$ M. Rubio-Roy, C. Corbella, J. García-Céspedes, M. C. Polo, E. Pascual, J. L. Andújar, and E. Bertran, Diamond Relat. Mater. 16, 1286 (2007).

${ }^{28}$ A. Pastol and Y. Catherine, J. Phys. D 23, 799 (1990).

${ }^{29}$ T. Wallendorf, S. Marke, and R. Bandorf, 49th SVC Annual Technical Conference Proceedings, 2006 (unpublished), p. 413.
${ }^{30}$ M. Bauer, T. Schwarz-Selinger, W. Jacob, and A. von Keudell, J. Appl. Phys. 98, 073302 (2005).

${ }^{31}$ H. Sugai and H. Toyoda, J. Vac. Sci. Technol. A 10, 1193 (1992).

${ }^{32}$ D. Gahan, B. Dolinaj, and M. B. Hopkins, Rev. Sci. Instrum. 79, 033502 (2008).

${ }^{33}$ N. Hershkowitz, in Plasma Diagnostics, edited by O. Auciello and D. L. Flamm (Academic, New York, 1989), Vol. 1, p. 113.

${ }^{34}$ V. I. Demidov, S. V. Ratynskaia, and K. Rypdal, Rev. Sci. Instrum. 73, 3409 (2002).

${ }^{35}$ D. Gahan, B. Dolinaj, and M. B. Hopkins, Plasma Sources Sci. Technol. 17, 035026 (2008).

${ }^{36}$ C. Corbella, M. C. Polo, G. Oncins, E. Pascual, J. L. Andújar, and E. Bertran, Thin Solid Films 482, 172 (2005).

${ }^{37}$ H. Bäcker and J. W. Bradley, Plasma Sources Sci. Technol. 14, 419 (2005).

${ }^{38}$ F. Richter, Th. Welzel, Th. Dunger, and H. Kupfer, Surf. Coat. Technol. 188-189, 384 (2004)

${ }^{39}$ A. Hallil and B. Despax, Thin Solid Films 358, 30 (2000).

${ }^{40}$ A. Joshi, S. A. Gangal, and S. K. Kulkarni, J. Appl. Phys. 64, 6668 (1988).

${ }^{41}$ R. G. Lacerda, V. Stolojan, D. C. Cox, S. R. P. Silva, and F. C. Marques, Diamond Relat. Mater. 11, 980 (2002).

${ }^{42}$ S. Peter, K. Graupner, D. Grambole, and F. Richter, J. Appl. Phys. 102, 053304 (2007).

${ }^{43}$ A. L. Alexandrov and I. V. Schweigert, Plasma Sources Sci. Technol. 14, 209 (2005)

${ }^{44}$ M. A. Lieberman and A. J. Lichtenberg, Principles of Plasma Discharges and Materials Processing (Wiley, New York, 2005).

${ }^{45}$ F. F. Chen, in Plasma Diagnostic Techniques, edited by R. H. Huddlestone and S. L. Leonard (Academic, New York, 1965), p. 113.

${ }^{46}$ F. F. Chen, Phys. Plasmas 8, 3029 (2001).

${ }^{47}$ H. Toyoda, H. Kojima, and H. Sugai, Appl. Phys. Lett. 54, 1507 (1989).

${ }^{48}$ M. Tuszewski and J. A. Tobin, Plasma Sources Sci. Technol. 5, 640 (1996).

${ }^{49}$ Z. Sternovsky, S. Robertson, and M. Lampe, Phys. Plasmas 10, 300 (2003).

${ }^{50}$ A. Karamcheti and Ch. Steinbrüchel, J. Vac. Sci. Technol. A 17, 3051 (1999).

${ }^{51}$ T. E. Sheridan, M. J. Goeckner, and J. Goree, J. Vac. Sci. Technol. A 9, 688 (1991).

${ }^{52}$ V. A. Godyak, R. B. Piejak, and B. M. Alexandrovich, J. Appl. Phys. 73, 3657 (1993).

${ }^{53}$ J. W. Bradley, H. Bäcker, P. J. Kelly, and R. D. Arnell, Surf. Coat. Technol. 142-144, 337 (2001).

${ }^{54}$ D. A. Glocker, J. Vac. Sci. Technol. A 11, 2989 (1993)

${ }^{55}$ A. Fridman and L. A. Kennedy, Plasma Physics and Engineering (Taylor \& Francis, New York, 2004).

${ }^{56}$ S. J. You, S. K. Ahn, and H. Y. Chang, Appl. Phys. Lett. 89, 171502 (2006).

${ }^{57}$ M. A. Lieberman and S. Ashida, Plasma Sources Sci. Technol. 5, 145 (1996).

${ }^{58}$ X. Tang and D. M. Manos, Plasma Sources Sci. Technol. 8, 594 (1999).

${ }^{59}$ H. Yasuda and T. Hirotsu, J. Polym. Sci., Polym. Chem. Ed. 16, 743 (1978).

${ }^{60}$ Y. S. Yeh, I. N. Shyy, and H. Yasuda, J. Appl. Polym. Sci.: Appl. Polym. Symp. 42, 1 (1988).

${ }^{61}$ D. Hegemann, M. M. Hossain, E. Körner, and D. J. Balazs, Plasma Processes Polym. 4, 229 (2007) 
${ }^{62}$ D. Hegemann, C. Oehr, and A. Fischer, J. Vac. Sci. Technol. A 23, 5 (2005).

${ }^{63}$ M. Bauer, T. Schwarz-Selinger, H. Kang, and A. von Keudell, Plasma Sources Sci. Technol. 14, 543 (2005).

${ }^{64}$ D. Hegemann, Thin Solid Films 515, 2173 (2006).

${ }^{65}$ C. Beneking, J. Appl. Phys. 68, 4461 (1990).
${ }^{66}$ V. A. Godyak, R. B. Piejak, and B. M. Alexandrovich, IEEE Trans. Plasma Sci. 19, 660 (1991).

${ }^{67}$ S. J. You, H. C. Kim, C. W. Chung, H. Y. Chang, and J. K. Lee, J. Appl. Phys. 94, 7422 (2003).

${ }^{68}$ S. J. You, S. K. Ahn, and H. Y. Chang, Surf. Coat. Technol. 193, 81 (2005). 